\title{
Surface plasmon investigations by STEM-EELS mapping of Au/Ni nanoparticles on STO
}

Thomas Aarholt ${ }^{1}$, Kevin Both ${ }^{2}$, Vilde Reinertsen ${ }^{2}$ and $\varnothing y s t e i n$ Prytz $^{2}$

${ }^{1}$ University of Oslo, Norway, ${ }^{2}$ University of Oslo, United States

Metallic nanoparticles have traditionally had sharp absorption peaks between 1 and $5 \mathrm{eV}$ due to the excitation of surface plasmons. Localized surface plasmon resonance (LSPR) is the coherent oscillation of quasi-free electrons excited by the electromagnetic fields of a photon or fast electron1,2. Since the absorption peak energies are in the range of visible light, surface plasmons present a potential pathway for absorbing energy to drive photoelectrochemical reactions or contribute to photovoltaic applications.

For driving photoelectrochemical reactions, one difficulty is placing photoactive nanoparticles near the catalyst responsible for a chosen reaction, for example, water splitting. The ideal semiconductor for water splitting has a bandgap of $1.9-2.3 \mathrm{eV}$, although semiconductors in this range are seldom stable during photocatalytic water splitting3. Utilizing plasmonics in wide bandgap semiconductors is a promising alternative, avoiding the stability issue3. In this work, we present a STEM-EELS study of Au nanoparticles created by galvanic replacement of Ni nanoparticles grown by exsolution of a PLD-deposited thin-film of A-site excess strontium titanate (Sr1.07Ti 0.93Ni0.07O 3- $\delta$ ) (STO). STO is an indirect, wide bandgap (3.2 $\mathrm{eV}$ ) perovskite, studied as a promising photocatalyst for water splitting 4 . Ni can easily substitute the $\mathrm{Ti}$ in the STO, making it a suitable candidate for exsolution, while Au cannot substitute Ti5. Simultaneously, $\mathrm{Ni}$ nanoparticles barely show any plasmonic activities, while Au particles are second only to Ag nanoparticles6. The presented manufacturing technique results in nanoparticles of gold and nickel freestanding, but socketed, on the surface and embedded in the bulk, with diameters between 50 and 100 $\mathrm{nm}$, which are well-suited for plasmonic applications.

As a starting point, STEM-EELS was performed with a monochromated 300kV STEM probe with ZeroLoss Peak (ZLP) full-width at half-maximum of $110 \mathrm{meV}$ on an FEI Titan G2. A peculiarly shaped gold nanocluster was studied. After removing the ZLP, the spectrum image was linearly fitted with Gaussians centered at 1.46, 1.95, and $2.40 \mathrm{eV}$. The $2.40 \mathrm{eV}$ peak is a well-known Au surface plasmon, whereas the remaining two are investigated in conjunction with modeling approaches. An RGB composite of the fit results can be seen in figure 1. Spectra extracted from the coloured regions can be seen in figure 2.

The Research Council of Norway is acknowledged for the support to the Norwegian Center for Transmission Electron Microscopy (NORTEM, no. 197405/F50) and the NANO2021 researcher project FUNCTION(no. 287729). 

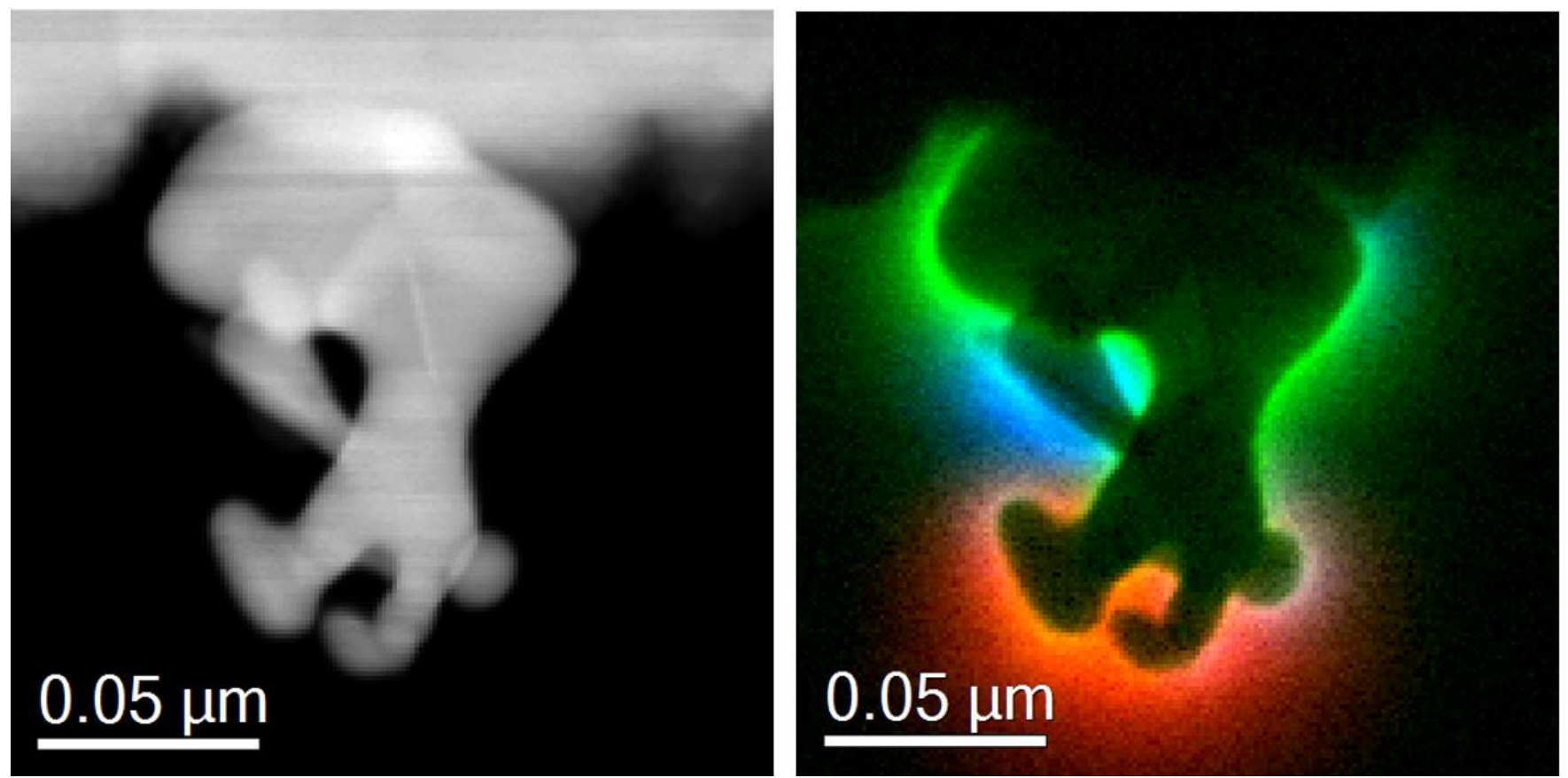

Figure 1. (left) HAADF image of a gold nanoparticle with irregular geometry. The top surface is a thinfilm of SrTiO3. (right) RGB composite image by mixing the resultant weights of fitting three gaussians on an EELS Spectrum Image taken around the nanoparticle. The surface plasmon peaks are coloured in the following manner: Red: $1.46 \mathrm{eV}$. Green: $2.4 \mathrm{eV}$. Blue: $1.95 \mathrm{eV}$.

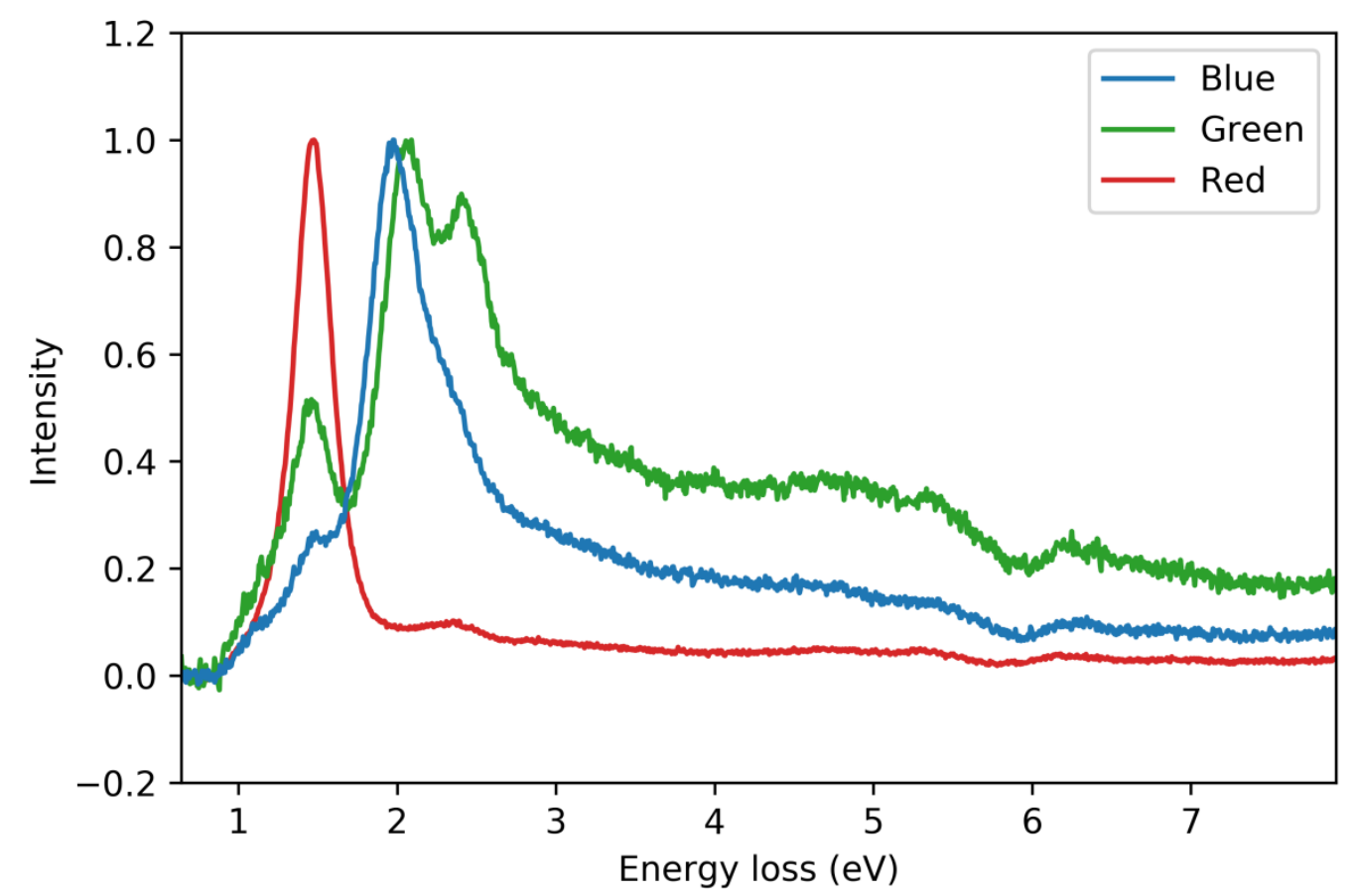

Figure 2. Spectra extracted from each of the coloured regions in the composite image in figure 1. 


\section{References}

1. Gao, Y., Murai, S., Shinozaki, K., Ishii, S. \& Tanaka, K. Aluminum for Near Infrared Plasmonics: Amplified Up-Conversion Photoluminescence from Core-Shell Nanoparticles on Periodic Lattices. Adv. Opt. Mater. 9, 2001040 (2021).

2. Koirala, K. P., Ge, J., Kalyanaraman, R. \& Duscher, G. Direct Detection of Highly Localized MetalMetal Interface Plasmons from Bimetallic Nanoparticles. Plasmonics (2021) doi:10.1007/s11468-02001345-x.

3. $\mathrm{Wu}, \mathrm{N}$. Plasmonic metal-semiconductor photocatalysts and photoelectrochemical cells: a review. Nanoscale 10, 2679-2696 (2018).

4. Phoon, B. L., Lai, C. W., Juan, J. C., Show, P.-L. \& Pan, G.-T. Recent developments of strontium titanate for photocatalytic water splitting application. Spec. Issue Adv. Hydrog. Prod. Technol. 44, 1431614340 (2019).

5. Neagu, D. Materials and microstructures for high temperature electrochemical devices through control of perovskite defect chemistry. (2013).

6. Kreibig, U. \& Vollmer, M. Optical Properties of Metal Clusters. (Springer Berlin Heidelberg, 2013). 